This is the peer-reviewed version of the following article: Chemistry - A European Journal 2015, 21, 3425-3434,

DOI: 10.1002/chem.201406110. Contents: Main text + supporting information.

The final form has been published at https://onlinelibrary.wiley.com/doi/abs/10.1002/chem.201406110.

This article may be used for non-commercial purposes in accordance with Wiley Terms and Conditions for Use of

Self-Archived Versions.

\title{
Towards ratiometric sensing of amyloid fibrils in vitro
}

\author{
Sonia Freire, ${ }^{[a]}$ Flor Rodríguez-Prieto, ${ }^{[b]}$ M. Carmen Ríos Rodríguez, ${ }^{[b]}$ J. Carlos Penedo, ${ }^{[c]}$ Wajih \\ Al-Soufi, ${ }^{[a]}$ and Mercedes Novo*[a]
}

\begin{abstract}
The aggregation of amyloid- $\beta$ peptide and its accumulation in the human brain has an important role in the etiology of Alzheimer's disease. Thioflavin T has been widely used as fluorescent marker of these amyloid aggregates. Nevertheless, its complex photophysical behavior, with strong wavelength dependencies of all its fluorescence properties, requires searching for new fluorescent probes. We propose the use of 2-(2'-hydroxyphenyl)imidazo[4,5b]pyridine (HPIP), which shows two emission bands and a rich excited-state behavior due to the existence of excitedstate intramolecular processes of proton transfer (ESIPT) and charge transfer (TICT). These properties determine a high sensitivity of HPIP fluorescence to its microenvironment
\end{abstract}

and cause a large differential fluorescence enhancement of the two bands upon binding to aggregates of the amyloid- $\beta$ peptide. Based on this behavior, a very sensitive ratiometric method is established for detection and quantification of amyloid fibrils, which can be combined with the monitoring of fluorescence anisotropy. Binding selectivity of HPIP is discussed on the basis of the apparent binding equilibrium constants of this probe to amyloid- $\beta$ (1-42) fibrils and to the non-fibrillar protein bovine serum albumin (BSA). Finally, an exhaustive comparison between HPIP and Thioflavin T is presented in order to discuss the sensitivity and specificity of these probes to amyloid aggregates and the significant advantages of the HPIP dye for quantitative determinations.

\section{Introduction}

The aggregation of misfolded peptides and proteins into toxic species that accumulate in various tissues and organs, leading to disruption of their normal function, is a major hallmark of many neurological degenerative disorders. These misfolded structures, so-called amyloids, are characterized by the formation of an anomalous cross- $\beta$-sheet conformation..$^{[1]}$ Among the different amyloidogenic sequences, amyloid- $\beta$ peptides $(A \beta)$ are attracting

[a] S. Freire, Prof. Dr. W. Al-Soufi, Prof. Dr. M. Novo

Department of Physical Chemistry

Faculty of Science, Campus Lugo

University of Santiago de Compostela

Campus Universitario s/n, 27001 Lugo (Spain)

Fax: $(+982824001$

E-mail:m.novo@usc.es

[b] Prof. Dr. F. Rodríguez-Prieto, Prof. Dr. M. C. Ríos Rodríguez Department of Physical Chemistry

Faculty of Chemistry and CIQUS, Campus Santiago de Compostela University of Santiago de Compostela

15782 Santiago de Compostela (Spain)

[c] Prof. Dr. J.C.Penedo

SUPA School of Physics and Astronomy

University of St Andrews

North Haugh, KY16 9SS (United Kingdom)

Supporting information for this article is available on the WWW under http://www.chemeurj.org/ or from the author. considerable interest due to their toxic role in the development of Alzheimer's disease, since they accumulate as insoluble aggregates among the neurons at the brain. ${ }^{[2]}$ These deposits are mainly formed by peptides of $40(\mathrm{~A} \beta 40)$ and 42 aminoacids (A 342$)$, which result from the $\beta$ - and $\gamma$-secretase cleavage of the amyloid precursor protein (APP). A 442 is less abundant than $A \beta 40^{[2-4]}$ however, it constitutes the more neurotoxic species due to its higher hydrophobicity, which results in faster aggregation rates at much lower concentrations than $A \beta 40 .{ }^{[5-7]}$

The most common marker used for the detection of amyloid fibrils is Thioflavin T (ThT), a fluorescent dye of the benzothiazole family that shows a dramatic fluorescence enhancement upon binding to amyloid aggregates. ${ }^{[8,9]}$ Several photophysical mechanisms have been proposed to explain this remarkable response. The main hypothesis postulates that ThT behaves as a molecular rotor, so that its fluorescence properties are determined by the extent of quenching of the locally excited state to a dark twisted intramolecular charge-transfer (TICT) state..$^{[9,10]}$ This quenching implies the twisting around the central $\mathrm{C}-\mathrm{C}$ single bond in the excited state ${ }^{[11]}$ and is therefore highly dependent on the viscosity of the medium. ThT molecules in aqueous solution are free to undergo the TICT process but in rigid media, as those potentially present as ThT binding sites in amyloid aggregates, the twisting process is hindered, increasing the fluorescence emission of the dye. In a recent contribution we confirmed this behavior of ThT as molecular rotor and unraveled some controversial features of its photophysical properties. ${ }^{[12]}$

Although the fluorescence enhancement of ThT has been widely used over the years to monitor amyloid aggregation, it is 
well known that ThT presents severe limitations that preclude its wider application as a quantitative method. For instance, we have recently shown a broad solvent-dependent distribution of ground-state conformations with very different photophysical behaviors and a strong wavelength dependency of all its fluorescence properties, which clearly limit its potential as an amyloid marker. ${ }^{[12]}$

Several alternative fluorophores for the determination of amyloid fibrils have been reported in recent years. For instance, $\mathrm{BSB}$ and $\mathrm{K} 114$, both derived from the structure of Congo-Red-like Chrysamine-G, have been used to recognize amyloid lesions and, in the case of $\mathrm{K} 114$, to follow fibril formation in solution through a quantitative fluorometric assay. ${ }^{[13-15]}$ Recently, new dyes based on benzothiazol and benzimidazole families have been designed to detect fibrillar $\beta$-lactoglobulin ${ }^{[16]}$ and also other compounds like tryptophanol were employed for monitoring $A \beta$ aggregation. ${ }^{[17]}$ Unfortunately, to date, most amyloid sensors have been developed around the concept of intensity changes of a single emission band to signal the aggregation process, a feature that intrinsically limits their application as quantitative tools for diagnostic applications. Moreover, the majority of sensors exhibit very little Stokes shift between the excitation and emission wavelengths, which also poses severe drawbacks for their use as bio-imaging agents. A potential solution to overcome these limitations is through the development of ratiometric probes. ${ }^{[18,19]}$ By following changes in the ratio of two emission bands as a function of analyte concentration, ratiometric probes provide a built-in reference to account for effects such as photobleaching or variations in sensor concentration, and also for environmental factors such as polarity, $\mathrm{pH}$ and changes in temperature, which may affect the intrinsic quantum yield of the probe and lead to incorrect interpretations. Interestingly, whereas a number of ratiometric sensors have been designed for ions ${ }^{[20-22]}$ or DNA, ${ }^{[23]}$ both in vitro and in vivo, only scarce examples exist for sensing protein aggregates. ${ }^{[24]}$

In this work, we demonstrate the potential of a novel marker, 2-(2'-hydroxyphenyl)imidazo[4,5-b]pyridine (HPIP), to act as a ratiometric probe for amyloid aggregation. HPIP is a member of the imidazole family, which is characterized by the presence, at physiological $\mathrm{pH}$ and temperature, of an equilibrium between two different fluorescently active hydrogen-bonded conformers (Scheme 1): $\mathbf{N}_{\text {inter }}(\mathrm{OH}$ group intermolecularly hydrogen bonded to the solvent) and $\mathbf{N}_{\text {intra }}$ (OH group intramolecularly hydrogen bonded to the imidazole nitrogen). It has been previously shown that the fluorescent properties of these so-called 'normal' (N) conformers ( $\mathbf{N}_{\text {intra }}$ and $\mathbf{N}_{\text {inter }}$ ) are significantly different and that their relative equilibrium populations are very sensitive to the environment. ${ }^{[25-28]}$ Excitation of the $\mathbf{N}_{\text {intra }}$ conformer yields a new zwitterionic species (tautomer $\mathbf{T}$, see Scheme 1) in the excited state, which emits with an extremely large red-shift $\left(\lambda_{\text {em }} \sim 480 \mathrm{~nm}\right)$ when compared to the normal-Stokes-shifted emission band of the $\mathbf{N}_{\text {inter }}$ conformer $\left(\lambda_{\mathrm{em}} \sim 380 \mathrm{~nm}\right)$. Simultaneous emission originating from the two species leads to the characteristic dualfluorescence behavior exhibited by HPIP and other members of the imidazole family. The hydrogen-bond ability of the solvent dictates the relative proportions of $\mathbf{N}_{\text {intra }}$ and $\mathbf{N}_{\text {inter }}$ conformers, and consequently, the intensity ratio between the two emission bands. But the solvent has also a profound influence on the quantum yield of the emissive species, facilitating further protontransfer processes of excited $\mathbf{N}_{\text {inter }}$ and a TICT process of excited

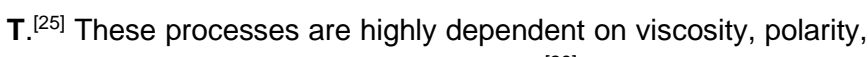
and acid-base properties of the medium, ${ }^{[29]}$ adding new factors to the sensitivity of HPIP fluorescence to the environment. All these facts suggest that HPIP may be a suitable probe for the development of bio-imaging agents. ${ }^{[18]}$
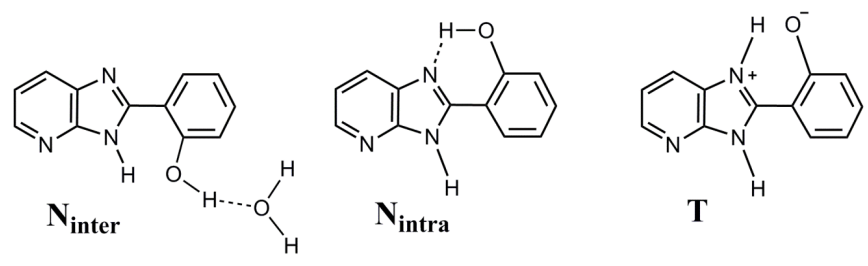

Scheme 1. Molecular structure of conformers and tautomers of HPIP. Structures shown are the normal forms $\mathbf{N}_{\text {inter }}(\mathrm{OH}$ group intermolecularly hydrogen bonded to the solvent) and $\mathbf{N}_{\text {intra }}(\mathrm{OH}$ group intramolecularly hydrogen bonded to the imidazole nitrogen), and the tautomer $\mathbf{T}$ (obtained by ESIPT after exciting $\mathbf{N}_{\text {intra }}$ ).

Here, we report a detailed characterization of the fluorescent behavior of HPIP in the presence of two proteins with different morphologies, the fibrillar aggregates of amyloid- $\beta$ and the globular bovine serum albumin (BSA). Our data have revealed a significant increase in the red-shifted emission of HPIP upon incubation with both types of proteins, which allows exploiting the dual emission of this probe for protein detection and quantification. A ratiometric quantity was successfully established to quantitatively determine protein concentration without interference from external parameters potentially present in ThT-based assays. This ratiometric assay should allow the direct quantification of amyloid fibers across different platforms and laboratories. Such standardization is crucial to resolve some of the discrepancies reported in the literature when using ThT as a probe. Moreover, HPIP exhibits a significant change in anisotropy between free and bound probe, absent when using ThT, which allows further characterization of the HPIP-protein interactions. We will also demonstrate that the affinity of HPIP for fibrillar A 342 is similar to that reported for ThT. ${ }^{[12]}$ In conclusion, the present study clearly indicates that HPIP outperforms ThT as a probe for amyloid- $\beta$ aggregates and suggests that a search among other members of the imidazole family may uncover additional ratiometric probes for amyloid sensing with further improved properties.

\section{Results and Discussion}

The main objective of this work is to explore the potential of HPIP as a ratiometric reporter of protein aggregates. Given the lack of available information regarding the fluorescence properties of HPIP in physiologically relevant aqueous conditions, we first carried out a photophysical study of HPIP in PBS buffer. Following the characterization of HPIP in this environment, we next report a detailed study on its fluorescence response as a protein probe using as model the fibril-like aggregates of amyloid- $\beta$ peptide and the globular protein BSA, respectively. These model systems were already used in a recently reported study with $\mathrm{ThT}^{\left[{ }^{[12]}\right.}$ so that the suitability of HPIP as fluorescent marker of amyloid fibrils can be directly compared with that of ThT. 


\section{Photophysical behavior of HPIP in physiologically relevant aqueous solutions}

The photophysical behavior of HPIP in different solvents has been thoroughly studied, ${ }^{[25-27]}$ but only scarce results have been published for HPIP in aqueous solution. ${ }^{[30]}$ Due to its low quantum yield in aqueous solution, most of the studies have been done with the dye dissolved in a mixture of water with $25 \%$ of ethanol to increase its solubility. However, we found that HPIP behavior in the presence of ethanol, even at small percentages, is quite different to that in water or aqueous buffer. Therefore, the first step of this work has been to investigate the photophysical behavior of HPIP in physiologically relevant aqueous solutions. For these studies, we chose as solvent a typical aqueous buffer solution used for protein samples (PBS buffer of $\mathrm{pH} 7.2$ ).

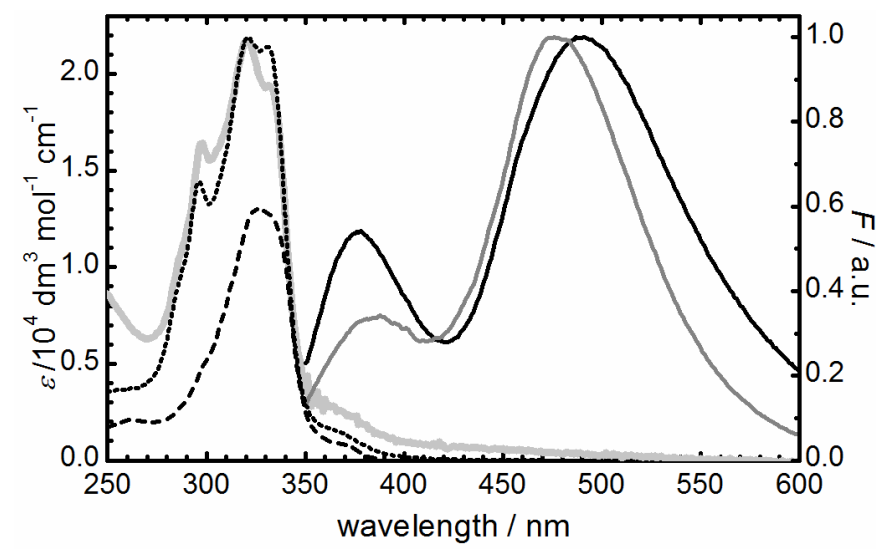

Figure 1. Absorption (gray thick solid line, left scale) and fluorescence excitation and emission spectra (right scale) of HPIP in PBS buffer of pH 7.2. Excitation spectra monitored at emission wavelengths of $400 \mathrm{~nm}$ (black dashed line) and $480 \mathrm{~nm}$ (black dotted line). Uncorrected emission spectrum measured with polarizers (dark gray solid line, total fluorescence intensity) and corrected emission spectrum without polarizers (black solid line).

The emission spectrum of HPIP in PBS buffer of $\mathrm{pH} 7.2$ (Figure 1) shows the characteristic long-wavelength emission band due to $\mathbf{T}^{\star}\left(\lambda_{\mathrm{em}} \sim 480 \mathrm{~nm}\right)$ and the short-wavelength emission band due to the normal form $\mathbf{N}^{*}\left(\lambda_{\text {em }} \sim 380 \mathrm{~nm}\right)$. The excitation spectrum recorded at $480 \mathrm{~nm}\left(\mathbf{T}^{\star}\right.$ band) corresponds to $\mathbf{N}_{\text {intra }}$ conformers, which undergo ESIPT upon excitation to yield $\mathbf{T}^{\star}$. This spectrum is very similar to the absorption spectrum (Figure 1). The excitation band monitored at $400 \mathrm{~nm}\left(\mathbf{N}^{\star}\right.$ band) is due to $\mathbf{N}_{\text {inter }}$ conformers, which are unable to give ESIPT. This band shows no vibrational structure and is red-shifted with respect to the absorption. This ability of this solvent, which indicates that $\mathbf{N}_{\text {intra }}$ is the main ground-state conformer of HPIP in water, despite the high hydrogen-bonding favors the $\mathbf{N}_{\text {inter }}$ conformers. Moreover, the weak shoulder observed at about $370 \mathrm{~nm}$ in the absorption spectrum is probably due to the existence of a minor amount of $\mathbf{T}$ in the ground state, in equilibrium with $\mathbf{N}_{\text {inter }}$ and $\mathbf{N}_{\text {intra }} \cdot{ }^{[31,32]}$ Scheme 2 summarizes the ground- and excited-state behavior of HPIP in PBS buffer of $\mathrm{pH}$ 7.2. This scheme includes the deprotonation of a fraction of $\mathbf{N}_{\text {inter }}$ molecules at the $\mathrm{OH}$ group upon excitation to give the anion, which is non fluorescent. ${ }^{[25]}$

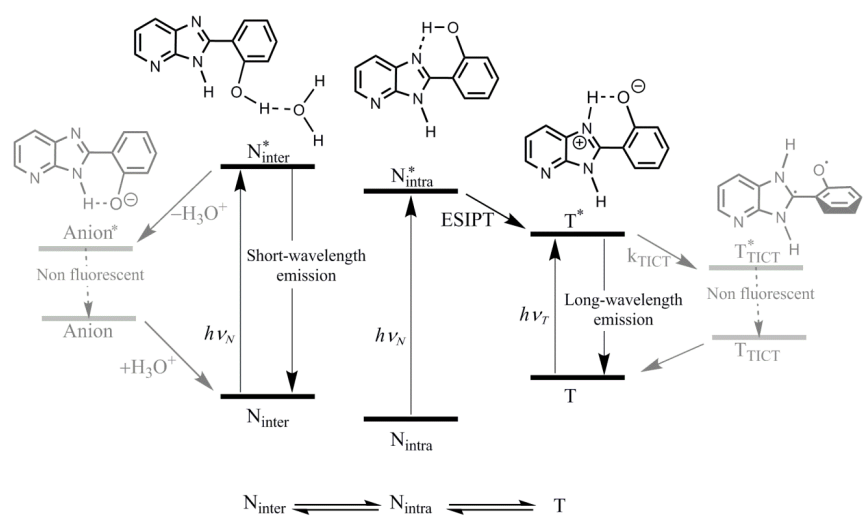

Scheme 2. Model proposed to explain the ground- and excited-state $\left(^{*}\right)$ behavior of HPIP. $\mathbf{N}_{\text {inter }}, \mathbf{N}_{\text {intra }}$ and $\mathbf{T}$ are in equilibrium in the electronic ground state. $\mathbf{N}_{\text {inter }}^{*}$ fluoresces at short wavelengths, whereas $\mathbf{T}^{\star}$ is responsible for the long wavelength emission of HPIP. $\mathbf{N}^{*}$ intra does not fluoresce because it undergoes an ultrafast ESIPT process to give the tautomer $\mathbf{T}^{\star}$. The fluorescence intensity of HPIP is dependent on the environment because it affects the effectivity of $\mathbf{N}_{\text {inter }}^{*}$ deprotonation and the intramolecular charge transfer of $\mathbf{T}^{\star}$, both of which produce nonemissive species.

For the proposed studies with proteins the emission spectra were recorded through polarizers. Besides the beneficial effect of preventing HPIP photodegradation (see experimental section), the use of polarizers allows determination of steady-state anisotropy and total fluorescence intensity from the same experimental data. Nevertheless, these emission spectra could not be corrected for instrumental factors (see experimental section), and therefore show significant differences both in shape and intensity with respect to the corrected emission spectrum recorded without polarizers (see Figure 1). However, the relative changes of the uncorrected spectra with protein concentration allow quantification of the dye-protein interaction in exactly the same way as the corrected spectra, provided the measurements are made in the same equipment. Additionally, we must emphasize that fluorescence anisotropy is independent of "instrumental corrections". Consequently, most of the measurements shown in this work have been done with polarizers, and the emission spectra will be shown without the "instrumental correction", unless otherwise noted. The fact that uncorrected spectra can be used for these studies represents an experimental simplification, useful for routine measurements.

\section{Spectral ratio $S R$ as a ratiometric magnitude sensitive to the surrounding medium}

The dual fluorescence of HPIP in aqueous solution makes it possible to establish a ratiometric method based on the quotient of the fluorescence intensities of the two emission bands. This method is highly recommended since the intensity ratio at two wavelengths is independent of dye concentration, and therefore provides an internal reference for the intensity measurements that the single emitters do not have. ${ }^{[18]}$

Initially, one would define a spectral ratio $S R$ as the quotient between the maximum intensities of the two bands. Nevertheless, we will define $S R$ as a valley-to-peak ratio. At the peak (average intensity at $490-495 \mathrm{~nm}$ ), the fluorescence of the tautomer $\mathbf{T}^{\star}$ reaches its maximum, whereas at the valley (average intensity at 
420-425 nm), the contribution of $\mathbf{N}^{*}$ prevails. The choice of these wavelengths to define $S R$ intends minimization of the following undesirable factors: (a) with an excitation wavelength of $330 \mathrm{~nm}$, the water Raman band is located at $373 \mathrm{~nm}$, which is close to the center of the short-wavelength emission band, and has an important contribution in comparison with the low fluorescence intensity of the dye in aqueous solution; (b) the influence of protein autofluorescence is greater at the wavelength range of the blue-shifted emission band; (c) residual photochemical decomposition of HPIP would have a stronger effect on the intensity of the short-wavelength emission maximum.

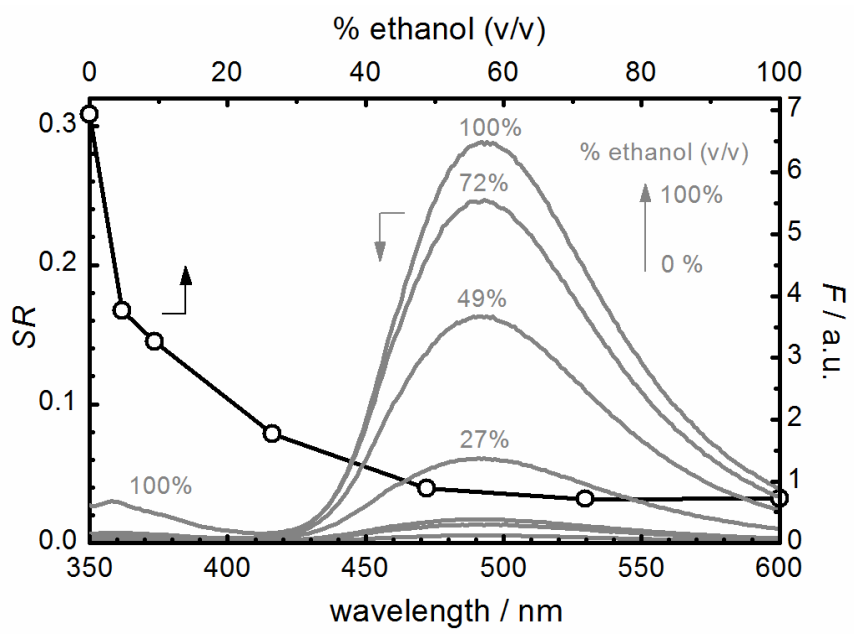

Figure 2. Corrected emission spectra (gray solid lines, lower and right scales, measured without polarizers) of HPIP in mixtures of PBS buffer with ethano $(0-100 \%(\mathrm{v} / \mathrm{v})$ ethanol/PBS mixture). The open circles represent the ratio $S R$ as a function of the volume percentage of ethanol (upper and left scales).

To test the ability of the spectral ratio $S R$ of HPIP to report variations in the properties of the surrounding environment, we have investigated the influence of added ethanol in the fluorescence properties of aqueous solutions of HPIP. As previously mentioned, ethanol percentages even as low as $25 \%$ induce a significant change in the fluorescence response of HPIP. Thus, the use of water/ethanol mixtures represents a suitable method to validate the spectral ratio $S R$ as reporter of the changes in HPIP environment.

The fluorescence intensity of HPIP significantly increases with the ethanol percentage in the mixtures ethanol/water, both at $\mathbf{N}^{\star}$ and $\mathbf{T}^{\star}$ emission bands (Figure 2). The fact that the intensities of both bands rise cannot be explained by a change in the ground-state equilibrium between $\mathbf{N}_{\text {inter }}, \mathbf{N}_{\text {intra }}$, and $\mathbf{T}$, and suggests that the fluorescence quantum yields of $\mathbf{N}^{*}$ and $\mathbf{T}^{\star}$ increase with the addition of ethanol. We propose that deprotonation of $\mathbf{N}_{\text {inter }}$ may be less efficient in ethanol than in pure water (see Scheme 2), resulting in a significantly higher emission of $\mathbf{N}^{\star}$ as the ethanol percentage is increased. With respect to $\mathbf{T}^{\star}$ emission, previous studies on HPIP ${ }^{[25]}$ showed that its fluorescence quantum yield in neutral solutions, mainly due to $\mathbf{T}^{\star}$, decreased from cyclohexane (0.31) to ethanol (0.14), and the value was even lower in water with $25 \%$ ethanol (0.03). A close look at the emission spectra of HPIP in aqueous solution with $27 \%$ ethanol and without ethanol (see Figure 2 ) reveals that the fluorescence quantum yield of HPIP in pure water must be much lower than 0.03 . $\mathbf{T}^{\star}$ probably undergoes in the excited state a large-amplitude rotational motion connected to a charge migration from the deprotonated phenol ring to the protonated imidazopyridine moiety (TICT process), leading to a nonfluorescent charge-transfer state (see Scheme 2) which is favored in aqueous solution. A similar twisted intramolecular charge-transfer process has been reported for the tautomer of various HPIP-related hydroxyarylbenzazoles. ${ }^{[25,29,33,34]}$

As shown in Figure 2, the value of the spectral ratio $S R$ for HPIP is highly sensitive to the environment of the dye. In aqueous medium, the ratio $S R$ has a value of approximately 0.3 , which drops abruptly with the addition of a small percentage of ethanol. When this percentage is increased, $S R$ decreases further until it reaches a constant value of about 0.03 in $100 \%$ ethanol. Therefore, the ratio $S R$ is a very sensitive parameter to monitor the HPIP environment and will be used in the following studies with proteins.

\section{Binding of HPIP to amyloid fibrils and BSA}

Once the spectral ratio $S R$ is validated as a sensitive quantity to probe the environment, we next used it to determine the apparent equilibrium constants for the binding of HPIP to A 442 fibrils and to BSA. We also used the fluorescence anisotropy $r$ as a second property sensitive to the binding of HPIP to proteins, as will be explained below.

Addition of $A \beta 42$ fibrils leads to a large fluorescence enhancement and a red shift of the long-wavelength emission band of HPIP, together with a moderate increase in the intensity of the short-wavelength emission band (Figure 3a). The fluorescence anisotropy shows also important changes with the concentration of fibrils, going from a constant value of 0.13 in buffer solution to higher values, which are wavelength dependent (Figure 3b). The anisotropy increase seems to be somewhat higher for the long-wavelength band assigned to $\mathbf{T}^{\star}$ than for the $\mathbf{N}^{*}$ band, although the anisotropy values at short wavelengths show distortions due to the effect of Rayleigh and Raman scattering.

We also studied the fluorescence of HPIP in the presence of the globular protein BSA, in order to compare its affinity and sensitivity to proteins of different morphologies and to examine the specificity of this probe for amyloid fibrils. Figure $1 \mathrm{~S}$ in the Supporting Information shows a fluorescence emission titration of HPIP in aqueous solution with BSA and the corresponding emission anisotropy spectra, all corrected by solvent and protein contribution (the emission spectrum of BSA at the highest concentration is also given to show the effect of its autofluorescence). Similarly to $A \beta 42$ fibrils, there is an increase of fluorescence intensity as the BSA concentration is raised, which is huge for the red-shifted emission band. Addition of BSA leads also to an anisotropy increase, which is very large for tautomer fluorescence (note that distortions due to Rayleigh and Raman scattering make it difficult to analyze the anisotropy values of the short-wavelength band). 

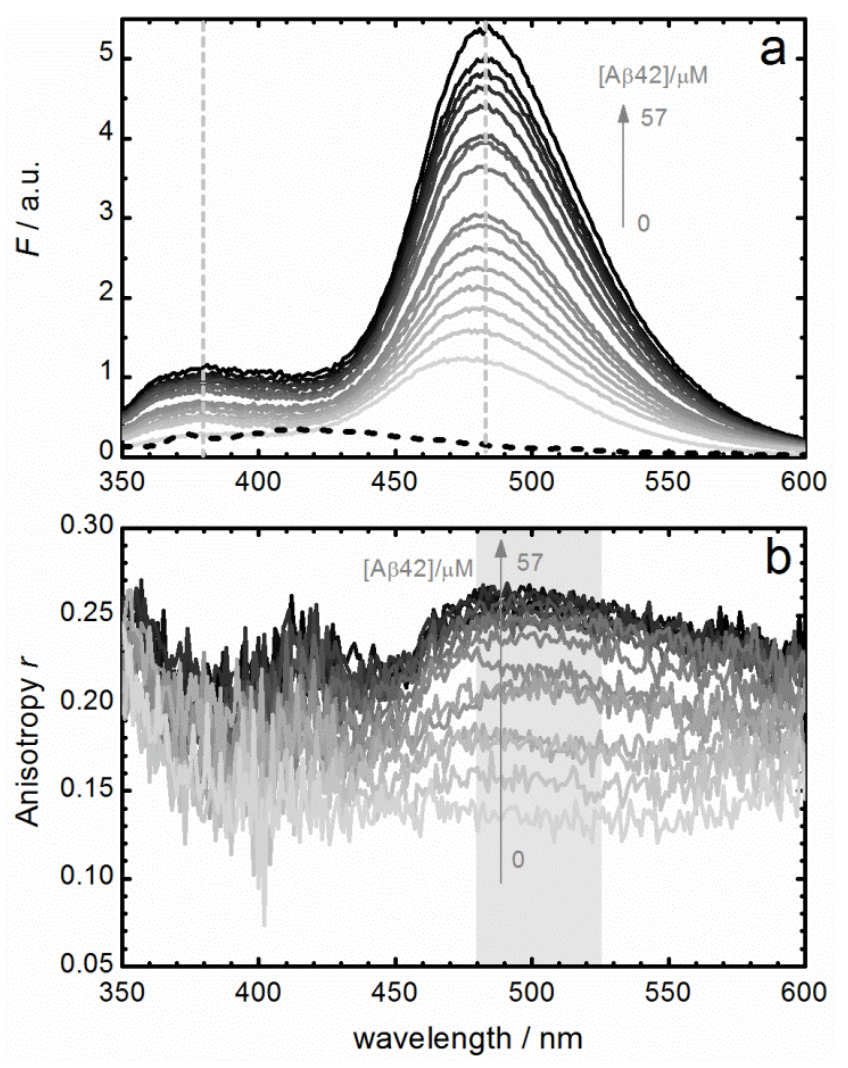

Figure 3. (a) Fluorescence emission spectra obtained by titration of HPIP in aqueous solution with $A \beta 42$ fibrils (solid lines). The dashed black line represents the amyloid autofluorescence, obtained for a solution of $A \beta 42$ fibrils at the highest concentration $(57 \mu \mathrm{M})$ without addition of HPIP. The vertical dashed gray lines indicate the positions of the two emission maxima. (b) Fluorescence anisotropy spectra of HPIP in aqueous solution with different concentrations of $A \beta 42$ fibrils. The wavelength interval used to determine the average values of anisotropy (from $480 \mathrm{~nm}$ to $525 \mathrm{~nm}$ ) is shown as gray rectangle. All spectra are corrected for the contribution of $A \beta 42$ autofluorescence and solvent (see experimental section).

The observed changes in the emission spectra of HPIP both with AB42 fibrils (Figure 3 ) and with BSA (Figure 1S in the Supporting Information) must be attributed to its binding to these proteins. This interaction seems to promote the formation of the excited tautomer $\mathbf{T}^{\star}$ and/or the increase of its fluorescence. The observed increase of fluorescence anisotropy at the tautomer band indicates that HPIP bound to the protein has a more constrained microenvironment than in aqueous solution. As a consequence, the nonradiative deactivation of the bound tautomer via TICT is hindered, exhibiting a large fluorescence enhancement. Similar but smaller effects are observed for the short-wavelength emission band assigned to $\mathbf{N}_{\text {inter }}^{*}$ conformer, indicating that this species is less quenched when bound to proteins (probably due to a lesser tendency to deprotonate than in water). The larger increase of the red-shifted emission band in BSA with respect to $A \beta 42$ fibrils, together with the higher anisotropy value reached, indicate that HPIP has a more constrained environment when bound to BSA.

Figure $2 S$ in the Supporting Information shows the excitation spectra for HPIP in PBS buffer and in the presence of a high concentration of $\mathrm{A} \beta 42$ fibrils or BSA. Apart from the contribution of protein autofluorescence at short wavelengths $(<280 \mathrm{~nm})$, the presence of proteins causes a shift to the red of the excitation spectrum monitored at $480 \mathrm{~nm}$. Moreover, the vibrational structure of the excitation spectra is amplified with respect to aqueous solution, probably due to the rigidity of the protein environment of the dye, as also confirmed by the anisotropy increase.
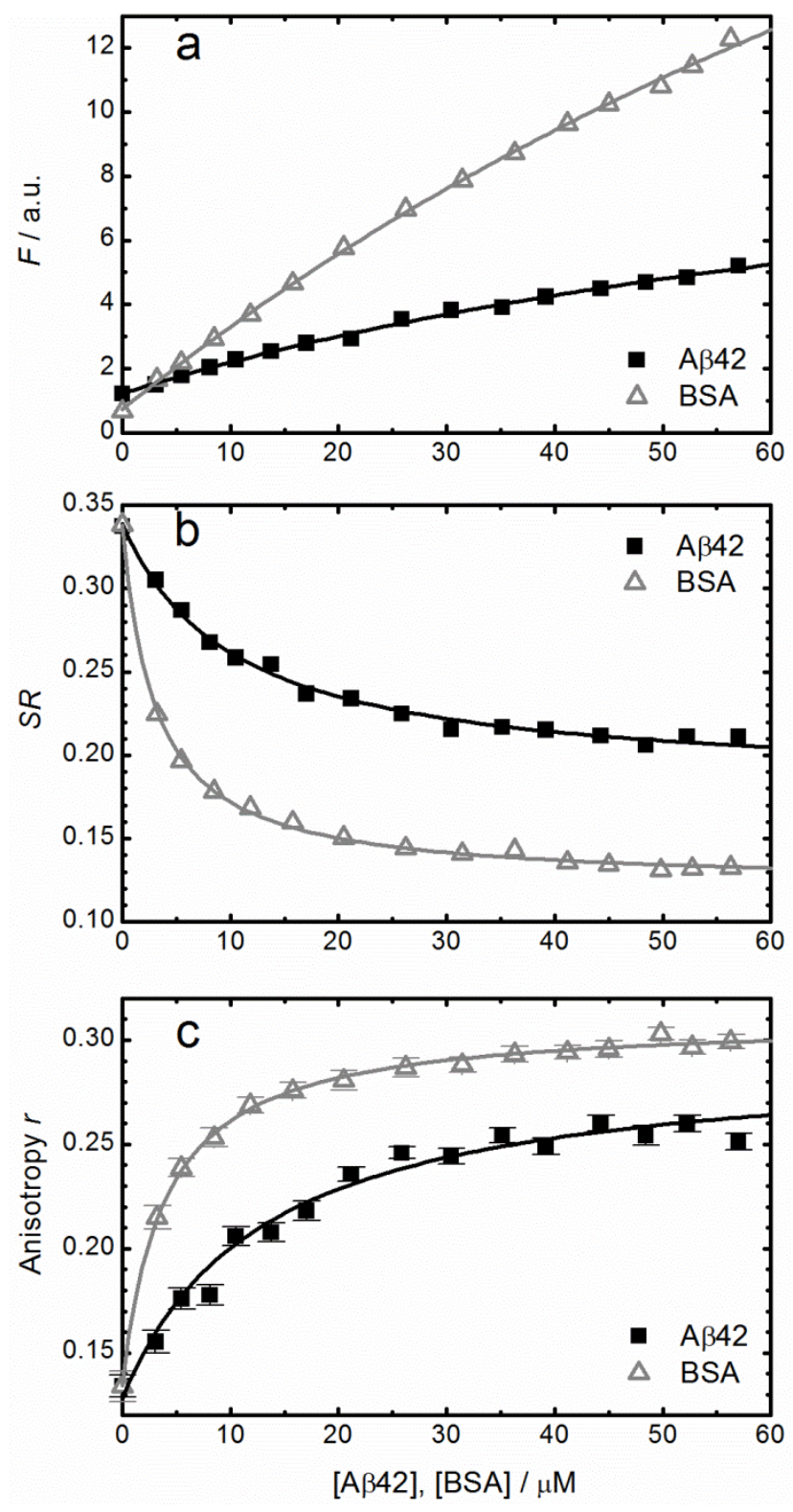

Figure 4. Plots of (a) fluorescence intensities at $490 \mathrm{~nm}$, (b) $S R$ ratio and (c) mean anisotropy $r(480 \mathrm{~nm}$ to $525 \mathrm{~nm})$ of HPIP against the concentration of A 442 (black squares) or BSA (gray triangles). All data are corrected for solvent contribution (essentially the intense Raman band) and protein autofluorescence. Solid lines are the fitted curves to intensity, $S R$ and $r$ data using equations (4), (5) and (6), respectively (see text for the fit parameters).

From the titration series (Figures 3 and $1 \mathrm{~S}$ in the Supporting Information), the dependencies of the different fluorescence properties of HPIP with protein concentration can be obtained. Figure 4 shows the experimental data for the two proteins of the 
fluorescence intensity $F$ at $490 \mathrm{~nm}$, close to the maximum of the long-wavelength band, the spectral ratio $S R$ as defined above, and the mean anisotropy $r$ of the long wavelength band (no suitable values can be determined for the short wavelength band due to the distortions caused by Rayleigh and Raman scattering). It is observed that all these three properties follow a typical binding profile (see equation (1) in the experimental section), varying from the value of the free dye to that of the dye bound to the protein. Nevertheless, both the spectral ratio $S R$ and the anisotropy approach constant values at protein concentrations where the fluorescence intensity just begins to deviate from a straight line. This indicates that the ratio SR and the anisotropy have greater sensitivity than fluorescence intensity and allow quantitative determinations with lower concentrations of protein.

Quantitative analysis of the variations of the three selected properties with the protein concentration is now performed on the basis of the binding equilibrium given in equation (1). The characteristic equilibrium constant $K$ is an apparent binding constant, since it is contributed by the different microscopic constants of each individual conformation and binding site. The fit functions for the fluorescence intensity at a certain wavelength, the spectral ratio $S R$ and the mean anisotropy $r$ are given in equations (4), (5) and (6), respectively. All these three functions have the same mathematical form and only differ in the meaning of the fit parameters. The binding constant $K$ can only be directly determined from the analysis of the absolute intensities, since it appears as the product $K_{\mathrm{q}}=K q_{b f}(\lambda)$ in the fit functions of $S R$ and $r$ (see equations (5) and (6)). Nevertheless, once the parameter $K_{\mathrm{q}}$ is determined, the binding constant $K$ can be calculated by dividing through the corresponding value of the brightness ratio $q_{b f}(\lambda)$. This ratio is defined as the quotient of fluorescence intensities of the bound dye and the free dye, and its value can be obtained from the "pure" spectra of the free dye and the dye bound to the protein, as explained below.

First we consider the analysis of the absolute fluorescence intensities, which allows determination of the binding equilibrium constant $K$ and the emission spectra of HPIP free and bound to the protein. The whole series of emission spectra (titrations as those shown in Figures $3 \mathrm{a}$ and $1 \mathrm{Sa}$ in the Supporting Information) were analyzed by the method of Principal Components Global Analysis (PCGA, see the experimental section), using equation (4) as fit function. PCGA confirmed that each series can be well reproduced by a linear combination of two independent components, which are assigned to free and bound HPIP. The fits yielded the values of the binding constant $K$ of HPIP to the proteins under study (Table 1) and the "pure" emission spectra of free and bound dye (Figure 5). It was checked that the spectrum obtained for the free dye agreed with that measured experimentally. The high quality of the fits can be appreciated in Figure $4 \mathrm{a}$ for the two proteins at a selected wavelength.

The values obtained for the binding equilibrium constant of HPIP to AB42 fibrils and to BSA are of the same order of magnitude, but somewhat larger for the former, indicating a higher affinity of HPIP to the fibrillar protein (Table 1). No data are available in the literature for the interaction of HPIP with $A \beta 42$ fibrils, but two studies have been recently published on the interaction of HPIP and analogues with BSA. ${ }^{[30,35]}$ The values reported for the binding constants of the different analogues to BSA are similar to ours, but the value determined for HPIP $\left(K=14.3 \times 10^{3} \mathrm{M}^{-1}\right)$ is about twice larger than that obtained in this study. Nevertheless, the method used in these publications is the linear Benesi-Hildebrand analysis, which involves strong assumptions with consequent inaccuracy of the quantitative results.

Table 1. Photophysical properties of HPIP in PBS buffer and bound to A 342 fibrils or to BSA: steady-state anisotropy $r$ (mean values in the wavelength range $480 \mathrm{~nm}$ to $525 \mathrm{~nm}$ ), spectral ratio $S R$, brightness ratio $q_{b f}(\lambda)$ determined from the "pure" fluorescence emission spectra, and binding constants $K_{q}$ and $K$ of HPIP to AB42 fibrils and to BSA.

\begin{tabular}{|lllllll|} 
& Method & $r$ & $S R$ & $q_{b f}(\lambda)$ & $\begin{array}{l}K_{q} / 10^{3} \\
\mathrm{~mol}^{-1} \mathrm{dm}^{3}\end{array}$ & $\begin{array}{l}K / 10^{3} \\
\mathrm{~mol}^{-1} \mathrm{dm}^{3}\end{array}$ \\
\hline PBS $^{[a]}$ & & $0.128 \pm 0.008$ & $0.345 \pm 0.004$ & - & - & - \\
A $^{342}$ & Intensity & - & - & $8.0 \pm 0.5^{[\mathrm{b}]}$ & - & $10 \pm 2$ \\
fibrils & Ratio SR & - & $0.185 \pm 0.004$ & $10.1 \pm 0.6^{[\mathrm{c}]}$ & $98 \pm 11$ & $10 \pm 1$ \\
& Anisotropy & $0.293 \pm 0.008$ & - & $10.0 \pm 0.6^{[\mathrm{d}]}$ & $78 \pm 16$ & $8 \pm 2$ \\
BSA & Intensity & - & - & $40 \pm 4^{[\mathrm{b}]}$ & - & $6.7 \pm 0.8$ \\
& Ratio SR & - & $0.124 \pm 0.001$ & $59 \pm 6^{[\mathrm{c}]}$ & $334 \pm 13$ & $5.7 \pm 0.6$ \\
& Anisotropy & $0.310 \pm 0.001$ & - & $59 \pm 6^{[\mathrm{d}}$ & $259 \pm 18$ & $4.4 \pm 0.5$ \\
\hline
\end{tabular}

[a] Values determined for free HPIP in the fits of anisotropy and $S R$ ratio experimental data. ${ }^{[b]}$ Value determined in the full range of the "pure" emission spectra $(350 \mathrm{~nm}$ to $600 \mathrm{~nm}) .{ }^{[c]}$ Value determined in the wavelength range from $490 \mathrm{~nm}$ to $495 \mathrm{~nm}$ of the "pure" emission spectra. [d] Value determined in the wavelength range from $480 \mathrm{~nm}$ to $525 \mathrm{~nm}$ of the "pure" emission spectra.

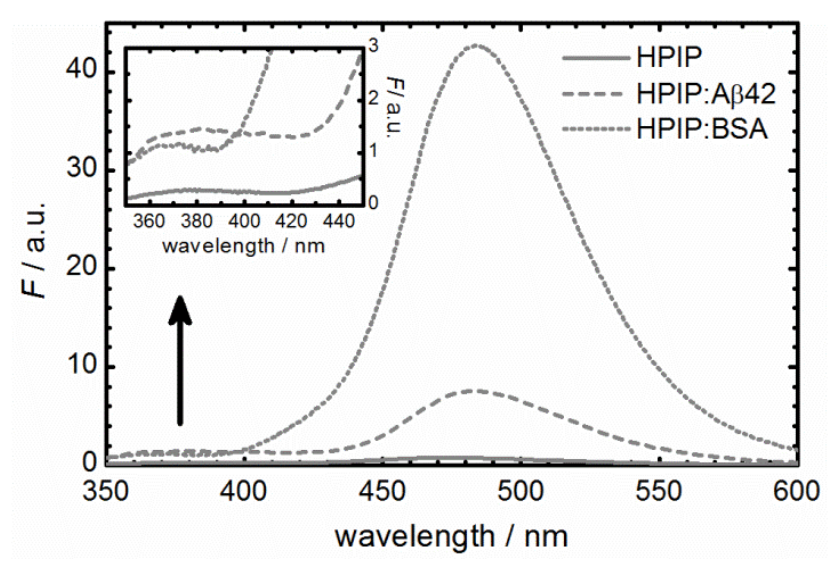

Figure 5. "Pure" fluorescence emission spectra obtained by global analysis of the corresponding titration series of emission spectra of HPIP with A $\beta 42$ fibrils and BSA: HPIP in PBS buffer (gray solid line), HPIP bound to A 342 fibrils (gray dashed line) and HPIP bound to BSA (gray dotted line). The spectra are corrected for the contributions of protein autofluorescence and solvent. The inset shows an enlargement of the same graph in the wavelength range from $350 \mathrm{~nm}$ to $450 \mathrm{~nm}$ in order to observe the changes in the short-wavelength band.

Comparison of the "pure" emission spectra of HPIP obtained in the analysis confirms the stronger tautomer fluorescence when the dye is bound to proteins and more significantly to BSA (Figure 5). The short-wavelength emission band of bound HPIP (see inset in Figure 5) exhibits a similar fluorescence enhancement for the two proteins. This result can be attributed to the reduction of the fluorescence quenching process that excited experiences in 
water due to the dissociation to give the non-fluorescent anion (see Scheme 2). This process is probably not efficient for HPIP bound to the protein due to the lack of an appropriate proton acceptor. Also a red shift of about $10 \mathrm{~nm}$ and a narrowing of the long wavelength emission band is also observed for HPIP bound to proteins with respect to the free dye (see normalized spectra in Figure $3 S$ in the Supporting Information). These changes suggest that the molecules of HPIP bound to A 342 fibrils or to BSA are restricted to certain conformations of the tautomer $T$ with very uniform fluorescence properties.

Quantitative analysis of the ratio $S R$ and the anisotropy $r$ was performed by non-linear fit of equations (5) and (6), yielding the apparent binding constant $K_{q}$ and the limiting values of $S R$ and $r$ for the free dye and the dye bound to protein (Table 1). The value of $K_{q}$ is larger when HPIP binds to BSA, suggesting a higher affinity of the dye to this protein, in contrast to the results obtained from the analysis of fluorescence intensities. The explanation of this apparent discrepancy is the value of the brightness ratio $q_{b f}(\lambda)$, which is included in the constant $K_{q}$. Its value is more than 5 times higher for HPIP bound to BSA than bound to amyloid fibrils, i.e., the fluorescence intensity is higher for the HPIP molecules bound to BSA than bound to $A \beta 42$ fibrils. Once $K_{q}$ is known, the binding equilibrium constant $K$ can be estimated using the values of $q_{b f}(\lambda)$ obtained from the "pure" emission spectra of free and bound HPIP (Figure 5) at the appropriate wavelength range (see Table 1). The values determined from the different properties for the binding constant $K$ agree well within their $95 \%$ confidence intervals, ensuring the consistency of the analysis.

From the values in Table 1, a great anisotropy increase is observed for HPIP upon binding to the proteins, which is about $10 \%$ higher for BSA, suggesting a more rigid microenvironment of the dye bound to the non-fibrillar protein. This is consistent with the higher fluorescence enhancement of the tautomer band for HPIP bound to BSA (Figure 5), since radiationless TICT deactivation would be more hindered than in amyloid fibrils. Consequently, also the limiting ratio $S R$ decreases more strongly in the case of BSA (Table 1) due to the higher fluorescence intensity of the tautomer band when HPIP is bound to this protein (Figure 5). Nevertheless, the limiting spectral ratios SR for HPIP bound to proteins do not reach values as low as in water-ethanol solutions of high alcohol percentages (see Figure 2), probably due to the simultaneous fluorescence enhancement of the two emission bands for the complexes of HPIP with proteins (see Figure 5).

Comparing the results obtained from the three selected properties: fluorescence intensity, spectral ratio and anisotropy, it is concluded that the binding of HPIP to a protein can only be well characterized from precise measurement and analysis of the absolute fluorescence intensities. Nevertheless, quantification of the amount of protein present in a sample is straightforward using either the ratio $S R$ or the anisotropy, once a calibration curve has been determined. The key advantages of these properties against absolute fluorescence intensity are their independency of the HPIP concentration and the much lower protein concentration required to obtain a suitable calibration curve.

The data shown in Figure 4 have been corrected for the contributions of solvent and protein fluorescence. The effect of these corrections on the three selected properties is shown in Figure 4S in the Supporting Information for the case of amyloid fibrils (analogous data are found for BSA). Since the solvent
Raman band appears at $373 \mathrm{~nm}$ and the proteins fluoresce around $420 \mathrm{~nm}$ (see emission spectra of A 42 fibrils and BSA in Figures $3 \mathrm{a}$ and $1 \mathrm{Sa}$, respectively), the correction has a strong effect on the intensity of the short-wavelength band. This is observed at first glance for the absolute fluorescence intensities, where the values at $390 \mathrm{~nm}$ are much more affected than those at $490 \mathrm{~nm}$ (Figure 4Sa). Also major differences are observed for the spectral ratio $S R$, as correction provides quite different limiting values due to its influence on the fluorescence intensity at the valley of the spectra, from $420 \mathrm{~nm}$ to $425 \mathrm{~nm}$. Instead, the mean anisotropy is almost not influenced by this correction because it is obtained from the long-wavelength emission band which is only slightly disturbed by protein autofluorescence. Nevertheless, the concentration dependency is analogous for the two types of data under the experimental conditions used in this work and therefore this data correction is not necessary, provided that consistent data are used for all data analysis.

\section{Comparison between HPIP and ThT as fluorescent markers of amyloid fibrils}

In this section we compare HPIP with ThT for their use as fluorescent probes in the detection and determination of amyloid fibrils.

Table 2. Photophysical properties of HPIP and ThT in PBS buffer and bound to fibrillar A 442 or to BSA: absorption maximum $\lambda_{a b}^{\max }$ (for ThT) or fluorescence excitation maximum $\lambda_{\text {exc }}^{\max }$ (monitored at $480 \mathrm{~nm}$, for HPIP), fluorescence emission maximum $\lambda_{e m}^{\max }$, steady-state anisotropy $r$, brightness ratio $q_{b f}(\lambda)$ and binding constant $K$ to $A \beta 42$ fibrils and BSA as determined by global analysis of the corresponding fluorescence titration series.

\begin{tabular}{|c|c|c|c|c|c|}
\hline $\operatorname{ThT}^{[a]}$ & $\lambda_{a b}^{\max } / \mathrm{nm}$ & $\lambda_{e m}^{\max } / \mathrm{nm}$ & $r^{[\mathrm{b}]}$ & $q_{b f}(\lambda)^{[c]}$ & $\begin{array}{l}K / 10^{3} \\
\mathrm{~mol}^{-1} \mathrm{dm}^{3}\end{array}$ \\
\hline PBS & 412 & 489 & $0.357 \pm 0.010$ & - & - \\
\hline $\begin{array}{l}A \beta 42 \\
\text { fibrils }\end{array}$ & 416 & 482 & $0.350 \pm 0.002$ & $1.6 \times 10^{3}$ & $5.85 \pm 0.07$ \\
\hline BSA & 415 & 483 & $0.378 \pm 0.004$ & $1.6 \times 10^{2}$ & $1.35 \pm 0.01$ \\
\hline HPIP & $\lambda_{a b}^{\max } / \mathrm{nm}$ & $\lambda_{e m}^{\max } / \mathrm{nm}$ & $r^{[b]}$ & $q_{b f}(\lambda)^{[c]}$ & $\begin{array}{l}K / 10^{3} \\
\mathrm{~mol}^{-1} \mathrm{dm}^{3}\end{array}$ \\
\hline PBS & 321,331 & 378,474 & $0.128 \pm 0.008$ & - & - \\
\hline $\begin{array}{l}\text { Aß42 } \\
\text { fibrils }\end{array}$ & 326,338 & 382,484 & $0.293 \pm 0.008$ & $8.0 \pm 0.5$ & $10 \pm 2$ \\
\hline BSA & 326,338 & 373,484 & $0.310 \pm 0.001$ & $40 \pm 4$ & $6.7 \pm 0.8$ \\
\hline
\end{tabular}

[a] Data taken from reference ${ }^{[12]}$ using an excitation wavelength of $410 \pm 10 \mathrm{~nm}$. ${ }^{[b]}$ Mean anisotropy determined in the ranges: $500-525 \mathrm{~nm}$ for ThT and 480-525 nm for HPIP. [c] Brightness ratio of the "pure" emission spectra: $440-650 \mathrm{~nm}$ for ThT and 350-600 nm for HPIP.

Table 2 shows a summary of the photophysical properties of HPIP and ThT in PBS buffer, bound to A 342 fibrils and bound to the non-fibrillar protein BSA. Because of the low solubility of HPIP, fluorescence excitation maxima are shown instead of absorption maxima. The fluorescence emission maxima and the mean anisotropy are also indicated. To compare the fluorescence intensity enhancement of the free and bound dyes, the brightness ratio $q_{b f}(\lambda)$ is determined in the full range of the pertinent "pure" fluorescence emission spectra. Finally, the binding equilibrium 
constants $K$ are given in order to discuss the specificity of ThT and HPIP to amyloid fibrils. These binding constants were obtained by global analysis of the corresponding series of fluorescence titrations. In the case of HPIP, these constants were also determined from the spectral ratio and the anisotropy variations but those obtained by global analysis were chosen to maintain the same determination method as in the case of ThT.

As fluorescent marker for proteins, the main advantage of ThT is its absorption maximum around $412 \mathrm{~nm}$, which is significantly red-shifted with respect to HPIP and therefore less sensitive to protein autofluorescence, as well as suitable for fluorescence microscopy. However, the fluorescence emission of ThT depends on the excitation-wavelength and shows a single emission band when excited at the absorption maximum. By contrast, fluorescence emission of HPIP does not depend on the excitation wavelength ${ }^{[25]}$ and exhibits dual emission. This feature allows a more sensitive detection and the quantitative determination of amyloid fibrils by using a ratiometric method, which is impossible with ThT.

In the case of ThT, two emission bands can be observed by exciting at short wavelengths around $330 \mathrm{~nm}$, corresponding to the free and the bound dye. Nevertheless, under these conditions, ThT shows very low fluorescence intensity and small changes upon binding to proteins. When ThT in a protein solution is excited at the absorption maximum, only the emission of the bound dye is observed since it is much more intense than that of the free dye. Therefore, only absolute measurements of fluorescence intensity are possible for ThT.

The brightness ratio $q_{b f}(\lambda)$ enables the comparison of the fluorescence enhancement of the two dyes upon binding to proteins, since it is defined as the ratio of fluorescence intensities of the bound dye with respect to the free dye. The value of $q_{b f}(\lambda)$ is about 200 times greater for ThT than for HPIP upon binding to amyloid fibrils, but only 4 times higher in the case of BSA (Table 2). This apparently convenient high contrast of ThT has however an adverse effect for quantitative determinations of the amyloid fibrils, since the fluorescent intensity of the free dye are almost undetectable and only the fluorescence coming from bound ThT is observed.

Regarding steady-state anisotropy, ThT shows similar values either when it is free in aqueous solution or bound to proteins (Table 2). These anisotropy values correspond to planar conformations that emit from the excited-stated without undergoing the TICT process. Therefore, this property of ThT cannot be used to detect proteins present in the solution. By contrast, the probe HPIP allows obtaining the anisotropy values of the free and bound dye in the long-wavelength emission band since they are associated to the tautomer emission. The steric constraints of HPIP when it is bound to proteins lead to a large increase of the tautomer mean anisotropy, together with a great fluorescence enhancement caused by a slower TICT deactivation.

The values of the binding equilibrium constants shown in Table 2 indicate that HPIP and ThT have comparable affinities to proteins. Nevertheless, the binding constant of HPIP to amyloid fibrils is approximately twice that of ThT. The binding constant of ThT to amyloids is about 4 times greater than to BSA, while HPIP has similar equilibrium constants for binding to both proteins. These results indicate that both dyes are very sensitive to fibrillar and non-fibrillar proteins, but they are not specific probes to identify fibrillar structures.

\section{Conclusions}

A new ratiometric fluorescence probe for the quantitative analysis of fibrillar A 342 and BSA proteins has been developed. The ESIPT-based dual-fluorescence probe HPIP showed a great enhancement of the long-wavelength emission band upon binding together with a large anisotropy increase. We demonstrated that the fluorescence intensity ratio of the two emission bands and the anisotropy can be successfully used for the determination of the mentioned proteins.

An exhaustive comparison of the behavior of HPIP with that of ThT demonstrated the great advantages of HPIP for sensitive and quantitative determination of amyloid fibrils. In addition, the photophysical properties of HPIP are not wavelength dependent as those of ThT.

Because of the advantages of HPIP, it should be considered improving its photochemical stability (see experimental section) by designing more stable derivatives with similar photophysical properties.

\section{Experimental Section}

\section{Materials}

2-(2'-hydroxyphenyl)imidazo[4,5-b]pyridine (HPIP) was prepared and characterized in our group. ${ }^{[25]}$ Bovine serum albumin (BSA) was purchased from Sigma-Aldrich, human amyloid- $\beta$ (1-42) (Aß42) from GenScript USA Inc and ethanol from Panreac. Phosphate buffered saline (PBS, $\mathrm{pH}=7.2,10 \mathrm{mM}$ phosphates and $140 \mathrm{mM} \mathrm{NaCl}$ ) was used for the preparation of the samples. All aqueous solutions were prepared using Milli- $Q$ water.

\section{Sample preparation}

HPIP stock solutions were prepared by stirring a small amount of solid in PBS for about $12 \mathrm{~h}$ until saturation and then filtering it using $0.45-\mu \mathrm{m}$ nylon filters. These stock solutions were used within a few days and diluted to the adequate concentration in each experiment $(1-4 \mu \mathrm{M})$. During the whole procedure, HPIP solutions were kept protected from light. In order to determine the real concentration of HPIP, a fluorescence calibration curve was performed for the HPIP concentration range $1-9 \mu \mathrm{M}$. To obtain the samples for calibration, a well-known amount of the dye was dissolved in ethanol. Then, an aliquot was taken and the ethanol was evaporated, redissolving the solute in buffer until the sample fluorescence remained stable. Fluorescence intensity of these samples showed a linear dependence with HPIP concentration. No dimerization or aggregation of HPIP was observed in the corresponding absorption spectra.

To prepare the samples of HPIP with different ethanol percentages (Figure 2), HPIP was first dissolved in ethanol and then diluted with the corresponding ethanol/buffer mixture. For the series shown in Figure 2, the concentration of HPIP was $5 \mu \mathrm{M}$.

Stock solutions of BSA and fibrillar $A \beta 42$ were freshly prepared for each experiment. The samples for the titrations were prepared by dilution of the corresponding protein stock solution containing the desired concentration of the dye with a solution of the same dye concentration. Amyloid concentrations mentioned in this work refer to the total concentration of amyloid monomers used in the preparation of the sample.

For the preparation of amyloid- $\beta$ fibrils, we followed established protocols. ${ }^{[36]}$ Firstly, potential aggregates that could be formed in the commercial peptide were broken up by hexafluoroisopropanol (HFIP). The amyloid was dissolved in HFIP at a concentration of $1 \mathrm{mg} / \mathrm{ml}$ and the solution was incubated during $1 \mathrm{~h}$ with occasional mixing and then shaken for about $20 \mathrm{~min}$. Next the solution was split up into several vials and the HFIP was removed under a stream of nitrogen Vials were transferred into a desiccator and vacuum was applied to remove the 
remaining traces of HFIP. The resulting dried aliquots of monomeric peptide were stored at $-20^{\circ} \mathrm{C}$.

Stock solutions of the amyloid were prepared by suspending the peptide aliquot in dried dimethyl sulfoxide (DMSO) with a final concentration of $1 \mathrm{mM}$ and shaking it for about $15 \mathrm{~min}$ in order to dissolve the peptide. To prepare amyloid- $\beta$ fibrils an aliquot of the DMSO stock solution was dissolved in PBS at $\mathrm{pH}$ 7.2. This sample was incubated at $37^{\circ} \mathrm{C}$ for 2 days under vigorous agitation.

\section{Photostability of HPIP}

The studies of the photophysical properties of HPIP in aqueous solution have evidenced some photoinstability of this dye, which mainly affects its short-wavelength fluorescence band. After preparing a HPIP stock solution, no significant changes were observed in its emission spectrum when a fresh aliquot was measured for several days. However, if a sample was continuously irradiated for some minutes, a significant increase in the fluorescence intensity of the short-wavelength emission band was detected, together with a sligh decrease of the long-wavelength emission band. This change was especially notable when using ultra-micro fluorescence cells where the sample volumes are very small (about $60 \mu \mathrm{L}$ for a $3 \times 3 \mathrm{~mm}$ cell). However, remixing the sample or refreshing it by addition of small amounts of fresh HPIP solution reduced the spectral changes significantly. Also the use of polarizers, with the consequently much lower irradiation intensity, minimized the problem. It was concluded that the observed spectral changes were due to a photochemical decomposition of HPIP that can be avoided by choosing suitable experimental conditions.

Because of the low quantum yield of HPIP in aqueous solution, it is necessary to use quite open slits in the excitation channel during fluorescence measurements. Also, ultra-micro fluorescence cells must be used for the smal volumes of the protein samples. Therefore, in order to prevent HPIP photodegradation, it was decided to perform the fluorescence measurements with polarizers because their presence reduces the light that reaches the sample in about 10 times. Furthermore, protein titrations were always performed by diluting the sample with fresh HPIP solution so that the effect of degradation is reduced.

\section{Absorption and fluorescence measurements}

Absorption spectra were recorded using quartz cells with path lengths of 3.00 and $10.0 \mathrm{~mm}$ in a Varian-Cary 100 spectrophotometer. All spectra were recorded using baseline correction with the corresponding solvent. Steady-state fluorescence measurements were performed using an Edinburgh-Instruments F900 spectrofluorometer equipped with a $450 \mathrm{~W}$ xenon lamp as excitation source. For anisotropy measurements, Glan-Thompson polarizers with a spectral range of $230-900 \mathrm{~nm}$ were incorporated. The excitation wavelength was $330 \mathrm{~nm}$, which is at the red flank of the absorption maximum to reduce the contribution of protein autofluorescence as much as possible. All measurements were performed at $25 \pm 1 \stackrel{\circ}{\circ}$.

Excitation and emission fluorescence spectra measured without polarizers were corrected for the non-ideal response of the instrument ("instrumental correction"). When polarizers were used, the spectra were not corrected because no appropriate correction factors were available. Furthermore, all spectra were corrected for the solvent contribution, in order to remove the Raman bands, which interfere for samples of low fluorescence intensity. The contribution of BSA and AB42 autofluorescence can also be subtracted to yield what we called "corrected" spectra. The influence of this correction on the data has been discussed above.

\section{Data analysis and fit functions}

Most data processing was done with the program OriginPro 8.5 (OriginLab Corporation, US). The series of emission spectra obtained in the titrations were analyzed using a program developed in our group which applies PCGA. ${ }^{37,38]}$ The first step of PCGA is the Principal Components Analysis (PCA), which yields the minimal number of spectral components that reproduce the experimental spectra. Global Analysis (GA) consists in the fit of the proposed theoretical model to the whole series of experimental spectra in order to estimate the model parameters and the "pure" spectra of the contributing species.
All reported uncertainties are based on the standard errors of the fitted parameters and do not take into account other sources of uncertainty, such as concentration errors.

A $1: 1$ binding equilibrium model between the free dye $\left(D_{f}\right)$ and the dye bound $\left(D_{b}\right)$ to the non-fluorescent protein (host, $H$ ) with a binding equilibrium constant $K$ (equation (1)) is used for the analysis of the experimental data. It must be noticed that $K$ is an apparent constant, since it comprises the contributions of the different microscopic constants of each individual conformation and binding site. Moreover, the interactions between the dye and the fibrillar amyloid are determined with reference to the total concentration of amyloid monomers and not to the fibrillar concentration in the sample, which is unknown. Nevertheless, the determination of the binding constant $K$ allows quantification of protein concentration and comparison of HPIP with other fluorescent probes.

$$
D_{f}+H \stackrel{K}{\longleftrightarrow} D_{b}
$$

Under the experimental conditions used (fixed dye concentration and excess of protein with respect to the total dye concentration, i.e. $[\mathrm{H}] \approx[\mathrm{H}]_{0}$ ) and assuming that excited-state association or dissociation do not take place, the following equations for the equilibrium concentrations of $D_{f}$ and $D_{b}$ can be easily deduced:

$$
\begin{aligned}
{\left[\mathrm{D}_{\mathrm{f}}\right] } & =\frac{[\mathrm{D}]_{0}}{1+K[\mathrm{H}]_{0}} \\
{\left[\mathrm{D}_{\mathrm{b}}\right] } & =\frac{[\mathrm{D}]_{0} K[\mathrm{H}]_{0}}{1+K[\mathrm{H}]_{0}}
\end{aligned}
$$

The fluorescence intensity at a certain wavelength, $F(\lambda)$, is the sum of the fluorescence intensities of the free and the bound dyes, which in turn are proportional to their concentrations. Then the following relation between the fluorescence intensity and the concentration of protein is deduced:

$$
F(\lambda)=\frac{F_{f}(\lambda)+F_{b}(\lambda) K[\mathrm{H}]_{0}}{1+K[\mathrm{H}]_{0}}
$$

where $F_{f}(\lambda)$ and $F_{b}(\lambda)$ are the limiting fluorescence intensities at the wavelength $\lambda$ of the dye free in aqueous solution and bound to the protein, respectively.

Equation (4) allows for the determination of the binding constant $K$ from fluorescence titrations. Nevertheless, this equilibrium constant can also be estimated from the spectral ratio $S R$ or the anisotropy $r$ obtained from the fluorescence titrations. The corresponding fit functions can be easily deduced using equation (4) and the definitions of these properties.

We define the ratio between the fluorescence intensities at two spectral wavelengths $\lambda_{1}$ and $\lambda_{2}$ of the same spectrum as the spectral ratio (SR). The variation of $S R$ with the concentration of protein is given by equation (5), where $S R_{f}$ and $S R_{b}$ are the limiting spectral ratios of the free dye and the dye bound to protein, respectively, and $q_{b f}\left(\lambda_{2}\right)$ is the brightness ratio between bound and free dye at the wavelength $\lambda_{2}$.

$$
S R=\frac{F\left(\lambda_{1}\right)}{F\left(\lambda_{2}\right)}=\frac{\frac{F_{f}\left(\lambda_{1}\right)}{F_{f}\left(\lambda_{2}\right)}+\frac{F_{b}\left(\lambda_{1}\right)}{F_{b}\left(\lambda_{2}\right)} \frac{F_{b}\left(\lambda_{2}\right)}{F_{f}\left(\lambda_{2}\right)} K[\mathrm{H}]_{0}}{1+\frac{F_{b}\left(\lambda_{2}\right)}{F_{f}\left(\lambda_{2}\right)} K[\mathrm{H}]_{0}}=\frac{S R_{f}+S R_{b} q_{b f}\left(\lambda_{2}\right) K[\mathrm{H}]_{0}}{1+q_{b f}\left(\lambda_{2}\right) K[\mathrm{H}]_{0}}
$$

For the steady-state anisotropy $r$ determined at a certain emission wavelength or wavelength range $\lambda$, equation (6) is obtained, where $r_{f}$ and $r_{b}$ are the limiting anisotropies of the free dye and the dye bound to protein, respectively, and $q_{b f}(\lambda)$ is the brightness ratio between bound and free dye at the wavelength $\lambda$.

$$
r=\frac{r_{f}+\frac{F_{b}(\lambda)}{F_{f}(\lambda)} r_{b} K[\mathrm{H}]_{0}}{1+\frac{F_{b}(\lambda)}{F_{f}(\lambda)} K[\mathrm{H}]_{0}}=\frac{r_{f}+r_{b} q_{b f}(\lambda) K[\mathrm{H}]_{0}}{1+q_{b f}(\lambda) K[\mathrm{H}]_{0}}
$$




\section{Acknowledgements}

S.F. thanks the Xunta de Galicia for a scholarship. The authors thank the European Regional Development Fund, the Ministerio de Ciencia e Innovación and the Xunta de Galicia for their financial support (CTQ2010-21369, CTQ2010-17835, GPC2013/052 and R2014/051). JCP thanks the RS MacDonald Charitable Trust for financial support. We thank Alfonso Brenlla for the synthesis of HPIP.

Keywords: 2-(2'-hydroxyphenyl)-imidazo[4,5-b]pyridine • amyloid fibrils $-\mathrm{BSA} \cdot$ proton transfer - charge transfer - ESIPT • TICT • fluorescent marker $\bullet$ ratiometric method $\bullet$ Thioflavin $\mathrm{T}$

[1] N. Melanie R, Methods 2004, 34, 151-160.

[2] F. M. LaFerla, K. N. Green, S. Oddo, Nat. Rev. Neurosci. 2007, 8, 499 509.

[3] Y. Chen, C. G. Glabe, J. Biol. Chem. 2006, 281, 24414-24422.

[4] D. M. Walsh, D. J. Selkoe, J. Neurochem. 2007, 101, 1172-1184.

[5] J. D. Harper, P. T. Lansbury Jr, Annu. Rev. Biochem. 1997, 66, 385-407.

[6] J. T. Jarrett, E. P. Berger, P. T. Lansbury, Biochemistry 1993, 32, 4693 4697

[7] C. Haass, D. J. Selkoe, Nat. Rev. Mol. Cell Biol. 2007, 8, 101-112.

[8] M. Groenning, J.Chem. Biol. 2010, 3, 1-18

[9] N. Amdursky, Y. Erez, D. Huppert, Acc. Chem. Res. 2012, 45, 1548 1557.

[10] A. A. Maskevich, V. I. Stsiapura, V. A. Kuzmitsky, I. M. Kuznetsova, O. I. Povarova, V. N. Uversky, K. K. Turoverov, J. Proteome Res. 2007, 6, 1392-1401.

[11] A. Srivastava, P. K. Singh, M. Kumbhakar, T. Mukherjee, S. Chattopadyay, H. Pal, S. Nath, Chem. Eur. J. 2010, 16, 9257-9263.

[12] S. Freire, M. H. de Araujo, W. Al-Soufi, M. Novo, Dyes Pigments 2014 110, 97-105.

[13] W. E. Klunk, M. L. Debnath, J. W. Pettegrew, Neurobiol. Aging 1995, 16 , 541-548.

[14] M. L. Schmidt, T. Schuck, S. Sheridan, M. Kung, H. Kung, Z. Zhuang, C Bergeron, J. S. Lamarche, D. Skovronsky, B. I. Giasson, Am. J. Pathol. 2001, 159, 937-943.

[15] A. S. Crystal, B. I. Giasson, A. Crowe, M. Kung, Z. Zhuang, J. Q. Trojanowski, V. M. Lee, J. Neurochem. 2003, 86, 1359-1368.

[16] K. Volkova, V. Kovalska, A. Balanda, R. Vermeij, V. Subramaniam, Y. L. Slominskii, S. Yarmoluk, J. Biochem. Biophys. Methods 2007, 70, 727 733.

[17] A. A. Reinke, G. A. Abulwerdi, J. E. Gestwicki, ChemBioChem 2010, 11, 1889-1895.

[18] A. P. Demchenko, J. Fluoresc. 2010, 20, 1099-1128.
[19] M. H. Lee, J. S. Kim, J. L. Sessler, Chem. Soc. Rev. 2014, In press.

[20] J. Wu, W. Liu, J. Ge, H. Zhang, P. Wang, Chem. Soc. Rev. 2011, 40, 3483-3495.

[21] Z. Liu, W. He, Z. Guo, Chem. Soc. Rev. 2013, 42, 1568-1600.

[22] H. Woo, Y. You, T. Kim, G. Jhon, W. Nam, J. Mater. Chem. 2012, 22 17100-17112

[23] J. Ueberfeld, D. R. Walt, Anal. Chem. 2004, 76, 947-952.

[24] D. A. Yushchenko, J. A. Fauerbach, S. Thirunavukkuarasu, E. JaresErijman, T. M. Jovin, J. Am. Chem. Soc. 2010, 132, 7860-1.

[25] A. Brenlla, M. Veiga, J. L. Pérez Lustres, M. C. Ríos Rodríguez, F Rodríguez-Prieto, M. Mosquera, J. Phys. Chem. B 2013, 117, 884-896.

[26] G. Krishnamoorthy, S. K. Dogra, J. Lumin. 2001, 92, 91-102.

[27] G. Krishnamoorthy, S. K. Dogra, J. Lumin. 2001, 92, 103-114.

[28] F. A. Chipem, G. Krishnamoorthy, J. Phys. Chem. A 2009, 113, 12063 12070.

[29] S. Rios Vazquez, M. C. Rios Rodriguez, M. Mosquera, F. RodriguezPrieto, J. Phys. Chem. A 2007, 111, 1814-1826.

[30] F. A. Chipem, S. K. Behera, G. Krishnamoorthy, Photochem. Photobiol. Sci. 2014, DOI: 10.1039/c4pp00099d

[31] F. Rodríguez Prieto, M. C. Ríos Rodríguez, M. Mosquera Gonzalez, M. A. Ríos Fernández, J. Phys. Chem. 1994, 98, 8666-8672.

[32] M. Mosquera, J. C. Penedo, M. C. Ríos Rodríguez, F. Rodríguez-Prieto, J. Phys. Chem. 1996, 100, 5398-5407.

[33] S. Rios Vazquez, M. C. Rios Rodriguez, M. Mosquera, F. RodriguezPrieto, J. Phys. Chem. A 2008, 112, 376-387.

[34] A. Brenlla, M. Veiga, M. C. R. Rodríguez, M. Mosquera, F. Rodríguez Prieto, Photochem. Photobiol. Sci. 2011, 10, 1622-1636.

[35] N. Dash, A. Mishra, G. Krishnamoorthy, J. Pharm. Biomed. Anal. 2013 $77,55-62$.

[36] W. B. Stine Jr, K. N. Dahlgren, G. A. Krafft, M. J. LaDu, J. Biol. Chem 2003, 278, 11612-11622.

[37] W. Al-Soufi, M. Novo, M. Mosquera, Appl. Spectrosc. 2001, 55, 630-636.

[38] W. Al-Soufi, M. Novo, M. Mosquera, F. Rodríguez-Prieto, in Reviews in Fluorescence 2009 (Ed: C. D. Geddes), Springer 2011, pp. 23-45.
Received: ((will be filled in by the editorial staff))

Revised: ((will be filled in by the editorial staff)) Published online: ((will be filled in by the editorial staff)) 
Entry for the Table of Contents (Please choose one layout only)

Layout 1:

\section{FULL PAPER}

A sensitive ratiometric method, based on a fluorescent probe undergoing excited-state intramolecular processes of proton transfer and charge transfer, is proposed for the detection and quantification of amyloid fibrils.

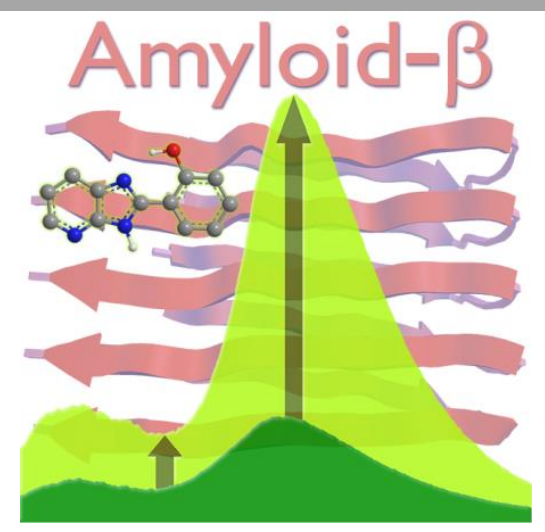

Subject Heading

Sonia Freire, Flor Rodríguez-Prieto, M. Carmen Ríos Rodríguez, J. Carlos Penedo, Wajih Al-Soufi, and Mercedes Novo*

$\mathbf{\square}-\mathbf{\square}$

Towards ratiometric sensing of amyloid fibrils in vitro 

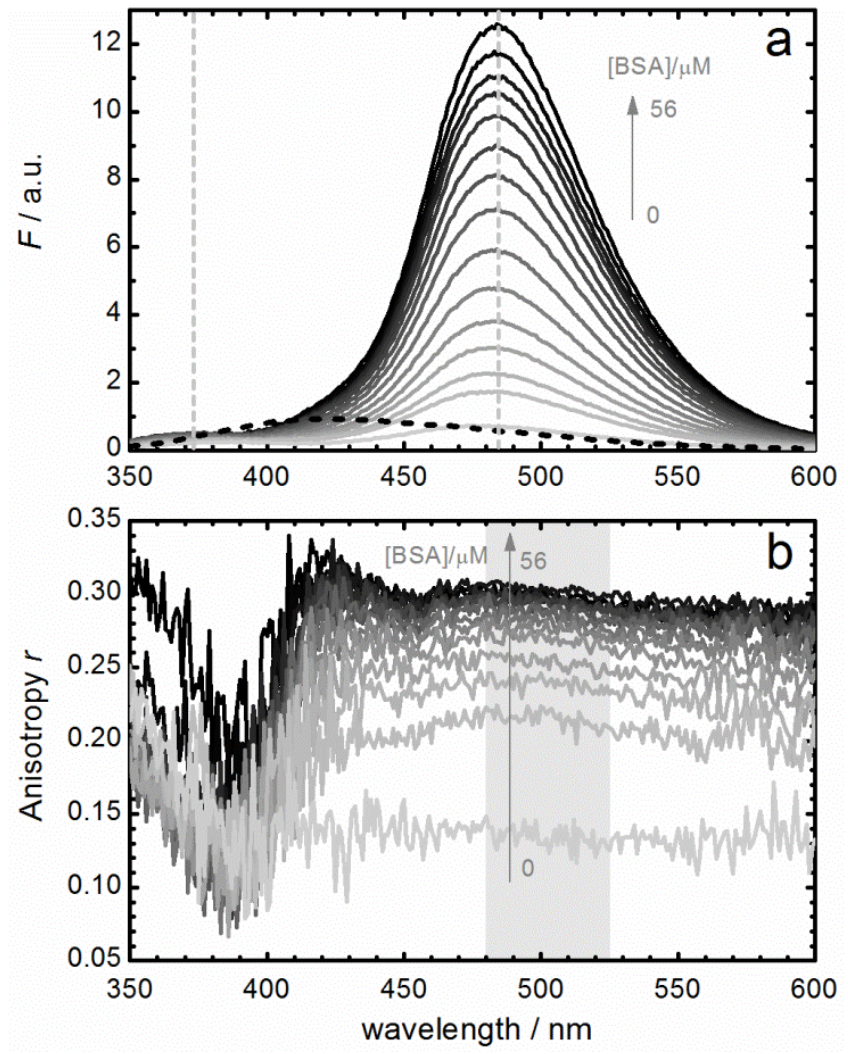

Figure 1S. (a) Fluorescence emission spectra obtained by titration of HPIP in aqueous solution with BSA (solid lines). The dashed black line represents the protein autofluorescence, obtained from a solution of BSA at the highest concentration $(56 \mu \mathrm{M})$, without addition of HPIP. The vertical dashed gray lines indicate the positions of the two emission maxima of HPIP. (b) Fluorescence anisotropy spectra of HPIP in aqueous solution with different concentrations of BSA. The wavelength interval used to determine the average values of anisotropy (from 480 to $525 \mathrm{~nm}$ ) is shown as gray rectangle. All spectra are corrected for the contribution of BSA autofluorescence and solvent (see experimental section). 


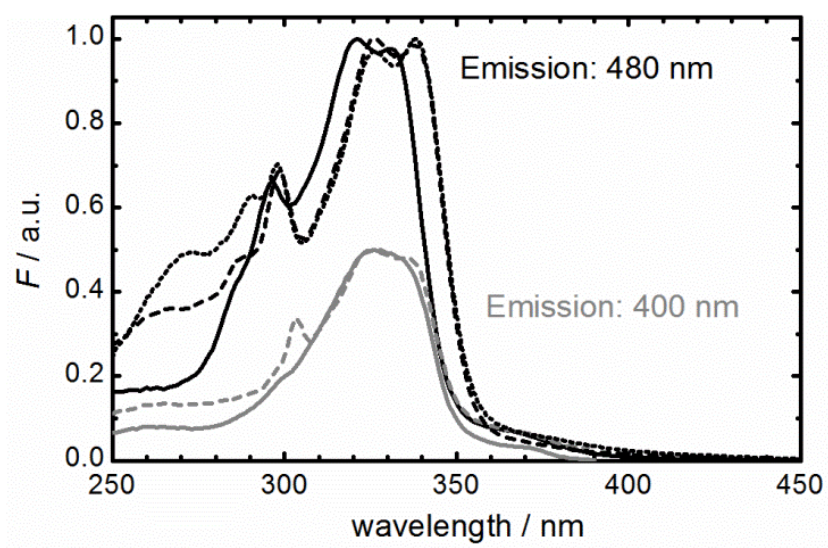

Figure 2S. Normalized fluorescence excitation spectra of HPIP in PBS buffer (solid lines), in the presence of A 442 fibrils $87 \mu \mathrm{M}$ (dashed lines) and in the presence of BSA $97 \mu \mathrm{M}$ (dotted lines). Excitation spectra monitored at $400 \mathrm{~nm}$ (gray lines, normalized to 0.5 at the maxima) and $480 \mathrm{~nm}$ (black lines, normalized to 1 at the maxima). For the sample of HPIP with BSA, the excitation spectrum monitored at $400 \mathrm{~nm}$ could not be measured due to the intense absorption band of the protein. Bands observed at wavelengths shorter than $280 \mathrm{~nm}$ in the samples with protein are due to protein fluorescence.

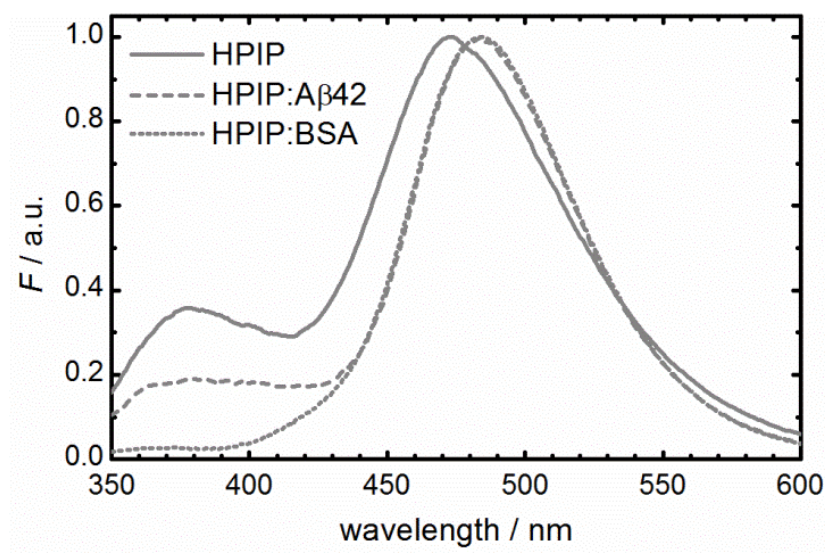

Figure 3S. Normalized "pure" fluorescence emission spectra obtained by global analysis (see materials and methods section in main manuscript) of the corresponding titration series of HPIP with A $\beta 42$ fibrils and BSA: HPIP in PBS buffer (gray solid line), HPIP bound to A $\beta 42$ fibrils (gray dashed line) and HPIP bound to BSA (gray dotted line). The spectra are corrected for the contributions of protein autofluorescence and solvent. 

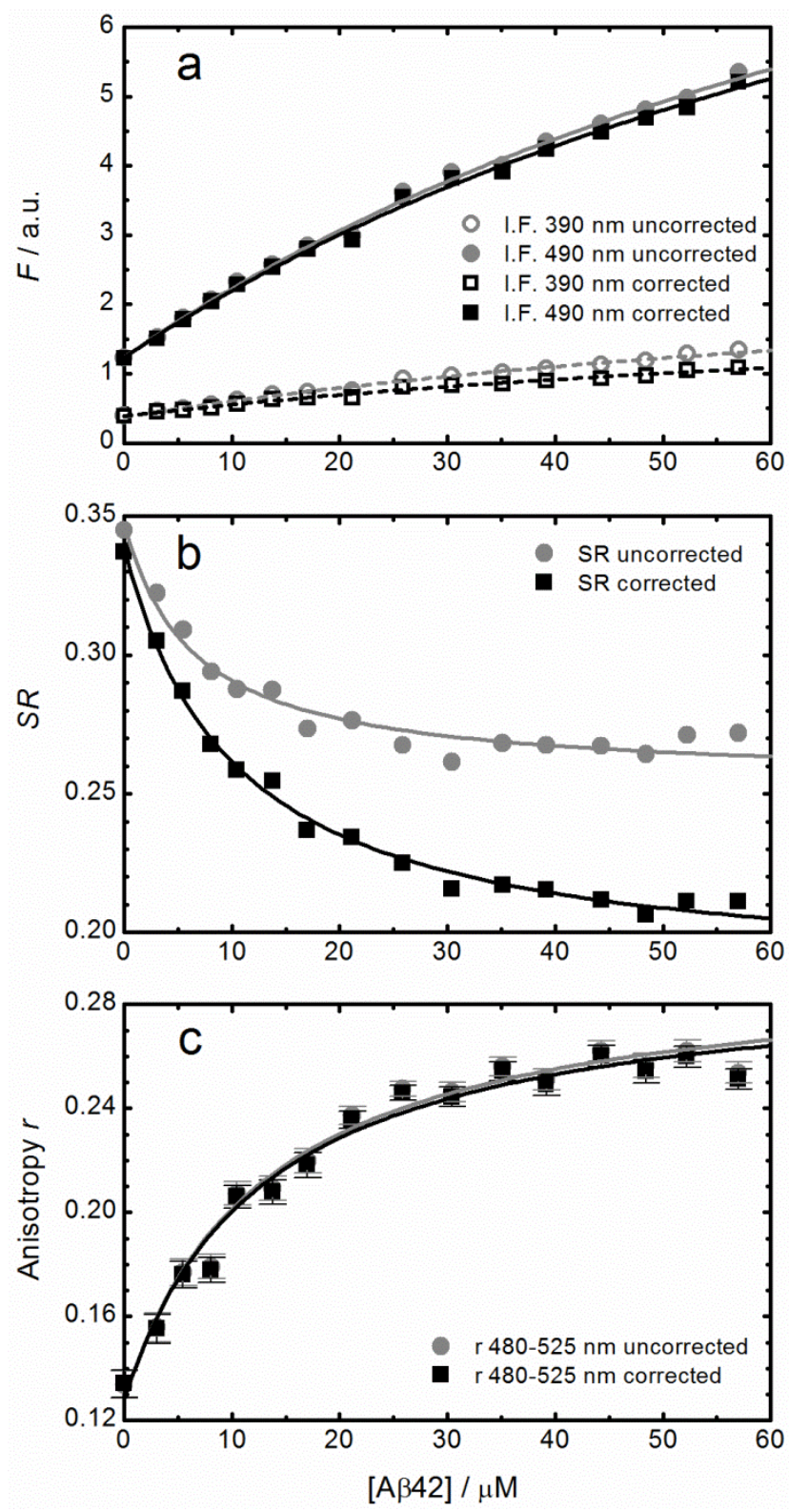

Figure 4S. Plots of (a) fluorescence intensities at $390 \mathrm{~nm}$ and $490 \mathrm{~nm}$, (b) $S R$ ratio and (c) mean anisotropy $r(480 \mathrm{~nm}$ to $525 \mathrm{~nm})$ of HPIP against A $\beta 42$ concentration. All three properties are shown as "uncorrected" (gray symbols) and "corrected" values (black symbols), where corrections for solvent contribution (essentially the intense Raman band) and for A $\beta 42$ autofluorescence were applied. Solid and dashed lines are the fitted curves to intensity, $S R$ and $r$ data using equations (4), (5) and (6), respectively (see text for the fit parameters). 